PENULTIMATE DRAFT - PLEASE CITE THE PUBLISHED VERSION

To appear in: Danks, D.; Ippoliti, E. (eds.), Building Theories, Springer, Cham.

\title{
The Noetic Account of Scientific Progress and the Factivity of Understanding
}

\author{
Fabio Sterpetti \\ Campus Bio-Medico University of Rome; Sapienza University of Rome \\ fabio.sterpetti@uniroma1.it
}

\begin{abstract}
There are three main accounts of scientific progress: 1) the epistemic account, according to which an episode in science constitutes progress when there is an increase in knowledge; 2) the semantic account, according to which progress is made when the number of truths increases; 3 ) the problem-solving account, according to which progress is made when the number of problems that we are able to solve increases. Each of these accounts has received several criticisms in the last decades. Nevertheless, some authors think that the epistemic account is to be preferred if one takes a realist stance. Recently, Dellsén proposed the noetic account, according to which an episode in science constitutes progress when scientists achieve increased understanding of a phenomenon. Dellsén claims that the noetic account is a more adequate realist account of scientific progress than the epistemic account. This paper aims precisely at assessing whether the noetic account is a more adequate realist account of progress than the epistemic account.
\end{abstract}

KEYWORDS: epistemology of modality; factivity; knowledge; noetic account; scientific progress; understanding

\section{Introduction}

Scientific progress is still one of the most significant issues in the philosophy of science today, since, as Chang states, neither "philosophers nor scientists themselves have been able to" settle this issue "to general satisfaction" (Chang 2007, p. 1). And this state of facts can be explained considering the "immense difficulty" of the topic.

Certainly, one of the main difficulties that arises in developing an account of scientific progress is to find the way to coherently take into account both of the two main characteristic features of science development: 1) theory change, which seems to unequivocally emerge every time we carefully analyse one of the main transitions in the historical development of science, and 2) the striking empirical success of our best scientific theories, which, according to scientific realism at least, i.e. the nowadays mainstream view in philosophy of science, cannot be adequately explained without 
referring to the (approximate) truth of our best theories (Niiniluoto 2015b; Chakravartty 2015).

Those two features of science development pull in different directions, hence the difficulty of giving a satisfying account of progress. Indeed, if a radical theory change occurs, the past theory should be regarded as false. But realists consider empirical success a good indicator of truth, and claim that successful theories are (approximately) true. So, if a theory enjoys empirical success, it should not undergo a really radical change and finally be dismissed as false. But history of science seems to provide examples of oncesuccessful theories that have nevertheless been successively dismissed, and for which it is not easy to demonstrate that there is some continuity between the new theories and the replaced ones. ${ }^{1}$ Did those past false theories constitute instances of genuine scientific progress? It depends on how we conceive of progress, namely on which requirements we think have to be fulfilled in order for a theory to be regarded as progressive. For example, if you take a realist stance on progress, you probably will require a theory to be (approximately) true in order to consider it an instance of progress.

In other words, the debate about scientific progress intersects the debate about scientific realism, i.e. the central topic in philosophy of science (Chakravartty 2015). For example, if we take the debate over scientific realism to be about what the aim of science is, ${ }^{2}$ then the relation between these two debates may be described as follows:

$\mathrm{X}$ is the aim of science just in case science makes progress when $\mathrm{X}$ increases or accumulates. (Dellsén, 2016, p. 73). ${ }^{3}$

Thus, determining the aim of science is relevant for the investigations on scientific progress, since it may give us a sort of criterion to determine whether progress occurred or not. For example, if in analyzing a historical case $\mathrm{C}$, we find that there has been an increase in $\mathrm{X}$ during $\mathrm{C}$, and $\mathrm{X}$ is the aim of science, we can conclude that $\mathrm{C}$ did constitute a case of progress. But even determining whether progress occurred or not is relevant in order to support or attack a specific view on what is the aim of science. For example, if we are able to show that in case $\mathrm{D}$, although it is uncontroversial that $\mathrm{D}$ constitutes progress, $\mathrm{X}$ did not increase, we can affirm that $\mathrm{X}$ cannot be taken to be the aim of science.

Recently, Dellsén (2016) proposed a new conception of scientific progress, the noetic account ${ }^{4}$ according to which an episode in science is progressive when there is an increase in scientists' understanding of a phenomenon. Dellsén's noetic account is mainly devoted to overcoming some of the inadequacies that afflict the epistemic account of

1 Cf. e.g. Niiniluoto 2015b, sec. 3.5: "many past theories were not approximately true or truthlike. Ptolemy's geocentric theory was rejected in the Copernican revolution, not retained in the form 'approximately Ptolemy'. Indeed, the progressive steps from Ptolemy to Copernicus or from Newton to Einstein are not only matters of improved precision but involve changes in theoretical postulates and laws."

2 Claiming that the debate over scientific realism is about what is the aim of science is just one of the many possible ways to define such debate that have been proposed so far (Chakravartty 2015), and it is used here just for illustrative purpose. Which characterization of scientific realism is the most adequate one does not impinge on the present article, since for any possible characterization of the debate over scientific realism, it is possible to define how this debate and the debate over scientific progress intersect each other in a way similar to the one presented here. For similar reasons, it is not relevant here to survey the different proposals that have been advanced on what is the aim of science.

3 See also Niiniluoto (2015b); Bird (2007).

4 On the reason why Dellsén named his view 'noetic', cf. Dellsén 2016, p. 72, fn. 2: “'Noetic' as in the Greek 'nous', which is often translated into English as 'understanding'.' 
progress developed by Bird (2007). Bird's proposal, notwithstanding the criticisms it received (see e.g. Rowbottom 2010; Saatsi 2016), has had wide resonance, since it revived a debate about scientific progress in which the two main accounts were the semantic account and the problem-solving account (see below, sec. 2), and it still remains one of the accounts of progress more congenial to scientific realists.

Dellsén claims that the noetic account is a more adequate realist account of scientific progress than the epistemic one. This article aims precisely at assessing whether the noetic account is a more adequate realist account of progress than the epistemic one. The article is organized as follows: the three main accounts of scientific progress are presented (sec. 2); then Dellsén's proposal is illustrated (sec. 3) and the concept of understanding is analysed in some detail (sec. 4); an argument is then proposed, which aims to assess whether the noetic account is an adequate realist view of progress by testing it against an uncontroversial yet problematic case of progress (sec. 5); it is pointed out that Dellsén's view of understanding is wanting, and it is examined whether the modality-based view of understanding recently proposed by Rice (2016) may represent a valid option to 'fix' the noetic account (sec. 6); since this option is shown to be available only at a very high cost, i.e. the commitment to some form of possible-worlds modal realism, it is examined whether another more promising realist account of modality, i.e. modalism, may be of use to support Dellsén's account (sec. 7); finally, some conclusions are drawn (sec. 8).

\section{The main accounts of scientific progress}

Three main accounts of scientific progress may be found in the extant literature: 1) the epistemic account, according to which an episode in science constitutes progress when there is an increase in knowledge (Bird 2007); 2) the semantic account, according to which progress is made when either the number of truths or the verisimilitude of a theory increases, depending on which variant of this account we are dealing with (Popper 1963; Niiniluoto 2015a); 3) and the problem-solving account, according to which progress is made when the number of problems that we are able to solve increases (Kuhn 1970; Laudan 1977).

Those conceptions mainly differ for the concept they take to be central in order to account for scientific progress: 1) the epistemic account is based on the concept of "knowledge"; 2) the semantic account is based on the concept of "truth" (or "verisimilitude", depending on which formulation of this approach we adopt); 3) the problem-solving account is based on the concept of "problem-solving". Being based on different concepts, those accounts lead to different outcomes in determining whether an episode has to be regarded as a genuine instance of scientific progress or not.

Each of the above described accounts of progress has received several criticisms in the last decades (see Niiniluoto 2015b for a survey). Each of these accounts, indeed, seems unable, relying on its proper criteria of progressiveness, to account for the progressiveness of some episodes that are instead usually taken to represent genuine cases of scientific progress. Finding out this sort of counterexample to the definition of progress given by rival accounts has been (and is still) the main business in the dispute over scientific progress.

To better illustrate this way of attacking rival accounts, and to present more in detail Bird's conception of progress, i.e. the conception that is mainly discussed by Dellsén, and from which Dellsén moves to develop his own proposal, we will describe some of the arguments given by Bird to support the inadequacy of the semantic conception (sec. 2.1) and the inadequacy of the problem-solving conception (sec. 2.2). 


\subsection{Bird's criticism of the semantic account}

Bird's defense of the epistemic view of progress, a view that can be traced back to Bacon (Bird 2007, p. 87, n. 1), begins with the consideration that this view is the one that better accounts for the intuitive meaning of scientific progress. Indeed, according to Bird, if we ask ourselves what scientific progress is, the intuitive answer is simple: science "makes progress precisely when it shows the accumulation of scientific knowledge; an episode in science is progressive when at the end of the episode there is more knowledge than at the beginning" (Bird 2007, p. 64).

In other words, in Bird's view, the epistemic conception is better than other accounts of progress, because those episodes in the history of science that according to our intuition represent genuine cases of progress are all cases in which an increase in knowledge occurs. At the same time, it is not always possible to observe in such cases a corresponding increase in the number of truths or in the number of problems that we are able to solve. So, the epistemic account fares better than other accounts of progress, and has to be preferred.

It is worth specifying that Bird takes knowledge to be not merely "justified true belief"; rather he takes it to be "true belief justified in a non-accidental way". In this way, he tries to avoid the possibility that an accidentally justified true belief may be regarded as an instance of genuine scientific progress:

we know that knowledge is not justified true belief, thanks to Gettier's counterexamples. Are then truth and justification jointly sufficient for a new scientific belief adding to progress? No, for precisely the same reasons that they do not add to knowledge. We may construct a Gettier style case of a scientific belief that is accidentally true and also justified [...]. Such a case will not be a contribution to progress. (Bird 2007, p. 72).

So, in Bird's view, while truth and justification are necessarily required for a new scientific belief to be regarded as an instance of scientific progress, that belief merely being true and justified is not sufficient to make it a genuine instance of scientific progress.

It is exactly following this line of reasoning that Bird develops his attack on the semantic view of progress. Indeed, in order to point out the implausibility of the semantic view, Bird asks us to consider the following scenario:

Imagine a scientific community that has formed its beliefs using some very weak or even irrational method $\mathrm{M}$, such as astrology. But by fluke this sequence of beliefs is a sequence of true beliefs. [...]. Now imagine that at time $t$ an Archimedes-like scientist in this society realises and comes to know that $\mathrm{M}$ is weak. This scientist persuades (using different, reliable methods) her colleagues that $\mathrm{M}$ is unreliable. [...]. The scientific community now rejects its earlier beliefs as unsound, realising that they were formed solely on the basis of a poor method. (Bird 2007, p. 66).

The problem for Bird is that if we adopt the semantic view, we should describe this case as follows: this community was experiencing progress until time $t$, because the number of truths held by the community was increasing, while after time $t$ the community started experiencing a regress, because it gave up the true beliefs previously accumulated, and so their number decreased. According to Bird, this way of representing this scenario is unacceptable, since it contradicts our intuition, according to which things go exactly the other way around: giving up beliefs produced by an unreliable method is a 
progressive episode, and should not be judged as regressive. On the other hand, merely accumulating beliefs through an unreliable method cannot represent a real instance of progress, despite the accidental truth of those beliefs.

Thus, according to Bird, the trend of progress growth matches the trend of knowledge growth, and not the trend of growth of the number of truths.

\title{
2.2. Bird's criticism of the problem-solving account
}

Bird (2007) elaborates a different scenario in order to criticize the problem-solving account of progress. He constructs his argument starting from an example made by Laudan (1977) in order to support the problem-solving account. ${ }^{5}$

Consider the following historical episode: Nicole d'Oresme and his contemporaries believed that hot goat's blood would split diamonds. Now, for Kuhn (1970) a puzzle is solved when a proposed solution is sufficiently similar to a relevant paradigmatic puzzlesolution. According to Laudan, a problem $\mathrm{P}$ is solved when the phenomenon represented by $\mathrm{P}$ can be deduced from a theory $\mathrm{T}$. But Laudan does not require that either $\mathrm{P}$ or $\mathrm{T}$ be true: "A problem need not accurately describe a real state of affairs to be a problem: all that is required is that it be thought to be an actual state of affairs" (Laudan 1977, p. 16). In this perspective, if Oresme's solution to the problem of splitting diamonds is sufficiently similar to a relevant paradigmatic solution in his historical context, according to Kuhn's standards, Oresme's solution provides a genuine solution to that problem, and thus, since progress amounts to problem-solving, it represents scientific progress. Moreover, if Oresme and his contemporaries were able to give a theory from which the splitting of diamonds by hot goat's blood is deducible, Oresme's solution represents scientific progress according to Laudan's standards as well.

The main problem with the problem-solving account of progress, according to Bird, is that both Kuhn and Laudan, i.e. the main supporters of this account, do not think of solving a problem as involving "knowledge", if knowledge is understood in the classical way as requiring (at least) truth. And this fact, in Bird's view, leads those who adopt this account to judge certain historical episodes or hypothetical scenarios in a way that contradicts our intuition about what scientific progress is. Consider again Oresme's scenario:

\begin{abstract}
imagine that some second scholar later comes along and proves at time $t$ by impeccable means that Oresme's solution cannot work. Whereas we had a solution before, we now have no solution. [...]. By Laudan and Kuhn's standards that would mark a regress. (Bird 2007, p. 69).
\end{abstract}

This way of representing the dynamic of scientific progress in this scenario is, according to Bird, unacceptable, since it contradicts our intuition, according to which things go exactly the other way round: since Oresme's solution was not a real solution, because it is demonstrably ineffective, the community was not experiencing any progress

5 Bird's presentation of the problem-solving account does not do justice to the theoretical richness of this approach. For reasons of space, we follow Bird. For some recent works that innovate the problem-solving view, see Cellucci (2013), Ippoliti (2014), Ippoliti, Cellucci (2016), who advocate the heuristic view, according to which knowledge increases when, to solve a problem, a hypothesis "is produced that is a sufficient condition for solving it. The hypothesis is obtained from the problem, and possibly other data already available, by some non-deductive rule, and must be plausible [...]. But the hypothesis is in its turn a problem that must be solved, and is solved in the same way" (Cellucci 2013, p. 55). 
until time $t$, while when at time $t$ such alleged solution was demonstrated to be ineffective by reliable means, that very fact constituted progress for the community.

In other words, according to Bird, while Oresme's solution "might reasonably have seemed to Oresme and his contemporaries to be a contribution to progress, it is surely mistaken to think that this $i s$ therefore a contribution to progress (Bird 2007, p. 69). In order to decide whether a solution is a real solution, and so whether it represents a genuine progressive episode, we have to refer to our own current standards on what is true in that domain, i.e. to our knowledge.

It is worth underlining here that Bird explicitly refers to our current knowledge in order to rebut Oresme's solution: indeed, he admits that it is reasonable to claim that such solution might have seemed to Oresme and his contemporaries, according to their system of beliefs, an instance of genuine knowledge, even if it is in fact not really an instance of genuine knowledge, according to our current knowledge.

Thus, according to Bird, the trend of progress growth matches the trend of knowledge growth, and not the trend of growth of the number of solved problems.

\section{The noetic account of scientific progress}

Recently, Dellsén (2016a) maintained that Bird's account of scientific progress is inadequate, and proposed the noetic account, according to which an episode in science constitutes progress when scientists achieve increased understanding of a phenomenon.

The peculiarity of this account with respect to its rivals is that it is based on the concept of "understanding", rather than on the concept of knowledge, truth, or problemsolving.

Generally speaking, "understanding" has to be intended here as it is usually intended in the debate over understanding and the value of knowledge that has spread in the last decade both in epistemology and philosophy of science. ${ }^{6}$ More precisely, according to Dellsén, "understanding" has to be construed as the ability of a subject to explain or predict some aspect of a phenomenon. In this perspective, an agent has some "scientific understanding of a given target just in case she grasps how to correctly explain and/or predict some aspects of the target in the right sort of circumstances" (Dellsén 2016a, p. 75). Thus, in Dellsén's view, "an episode in science is progressive precisely when scientists grasp how to correctly explain or predict more aspects of the world at the end of the episode than at the beginning" (Dellsén 2016a, p. 72).

Dellsén claims that, since the noetic account rests on the concept of understanding instead of the concept of knowledge, the noetic account fares better than the epistemic one, because it is able to account for two classes of events that the epistemic account is not able to account for, namely: 1) cases in which progress occurs, while no increase in knowledge occurs (sec. 3.1); and 2) cases in which an increase in knowledge occurs, while no progress is made (sec. 3.2).

\subsection{Progress without knowledge increase}

6 For a survey on the issue of understanding, see Baumberger, Beisbart, Brun (2017); de Regt, Leonelli, Eigner (2009). On the related issue of the value of knowledge, see Pritchard and Turri (2014) for a survey. With regard to the debate over understanding in epistemology, see Elgin (2007), (2009); Kvanvig (2003); Zagzebski (2001); with regard to the debate over understanding in philosophy of science, see de Regt (2009), (2015); de Regt and Gijsbers (2017); Mizrahi (2012); Khalifa (2011), Grimm (2006). 
In order to point out the inadequacy of the epistemic account, Dellsén (2016a) considers Einstein's explanation of Brownian motion in terms of the kinetic theory of heat, presented in one of his famous annus mirabilis papers, "On the Movement of Small Particles Suspended in a Stationary Liquid Demanded by the Molecular-kinetic Theory of Heat" (Einstein 1905/1956). Einstein's paper's first paragraph reads:

\begin{abstract}
In this paper it will be shown that according to the molecular-kinetic theory of heat, bodies of microscopically-visible size suspended in a liquid will perform movements of such magnitude that they can be easily observed in a microscope, on account of the molecular motions of heat. It is possible that the movements to be discussed here are identical with the so-called 'Brownian molecular motion'; however, the information available to me regarding the latter is so lacking in precision, that I can form no judgment in the matter. (Einstein 1905/1956, p. 1).
\end{abstract}

Dellsén maintains that if we adopt the epistemic account of scientific progress, we should conclude that Einstein's contribution does not represent a case of genuine progress. Indeed, the epistemic account rests on the concept of knowledge, and knowledge requires (at least) truth and justification. Since Einstein's information on Brownian motion was lacking, Einstein clearly did not have "the epistemic justification required to know that the movements in question were in fact real. Thus, the explanandum in Einstein's explanation of Brownian motion did not constitute knowledge for Einstein at the time" (Dellsén 2016a, p. 76). Moreover, the kinetic theory on which Einstein's paper rests was in 1905 a disputed theory, and many reputable scientists did not accept the existence of atoms at that time (Perrin's decisive results were published starting from 1908, see Perrin 1908). So, also the explanans in Einstein's explanation of Brownian motion did not constitute a clear case of knowledge when Einstein published his paper.

Given that according to the epistemic account, scientific progress occurs when an increase in knowledge occurs, since in this case it cannot be affirmed that an increase in knowledge occurred, because neither the explanans nor the explanandum were known at the time, we should conclude that Einstein's contribution to science did not constitute progress in 1905.

According to Dellsén, this way of evaluating this historical episode is unacceptable, since it is unable to accommodate our common intuitions on what constitutes scientific progress. For Dellsén, this case underlines the inadequacy of the epistemic account in dealing with those cases in which progress occurs even if this progress cannot be said to constitute an increase in knowledge at the time when it occurs.

On the contrary, according to the noetic account, in 1905 a remarkable "cognitive" progress occurred, even if there wasn't a simultaneous increase in scientific "knowledge", since an explanation of an (until then) unexplained phenomenon was proposed. And Einstein's explanation was able to integrate such a phenomenon into a wider set of already explained phenomena, making it coherent with background knowledge, and so increasing the intelligibility of the phenomenon. In other words, an increase in the understanding of Brownian motion occurred in 1905.

In order to defend the epistemic account, it may be objected that Einstein's contribution to scientific progress consisted in gaining the knowledge that the kinetic theory would explain Brownian motion, if the kinetic theory is true. In this view, "Einstein's achievement amounts to gaining a kind of hypothetical explanatory knowledge - knowledge of how a potential explanans would explain a potential explanandum if the explanans and explanandum are both true" (Dellsén 2016a, p. 77). Unfortunately, this option seems to be unavailable for the supporter of the epistemic account. Indeed, if achieving some hypothetical explanatory knowledge may constitute 
genuine progress for the epistemic account, then this account is no more able to rule out Oresme's solution to the problem of splitting diamonds. In this view, to make Oresme's solution an acceptable solution, it would have been sufficient for its supporters to provide some theory about the supposed relation between hot goat's blood and diamonds, arranged in such a way that if this theory and Oresme's solution are true, then such theory would explain the splitting of diamonds through hot goat's blood. This theory would be an instance of hypothetical explanatory knowledge, and so it should be taken to constitute progress. But Bird, as we have seen, explicitly denies that Oresme's solution may constitute an instance of scientific progress. It is important to stress that, in order to discriminate between Oresme's and Einstein's cases, taking into account whether a hypothetical explanatory knowledge has later been confirmed is not a workable criterion. Indeed, if hypothetical explanatory knowledge has to wait until it is confirmed to be regarded as a genuine instance of progress, then it is not distinguishable from ordinary knowledge, and we should maintain that progress occurs when knowledge is acquired, i.e. when confirmation occurs. But if this is the case, then the epistemic account would again be unable to claim that genuine progress occurred in 1905 thanks to Einstein's work. So, the supporter of the epistemic account faces a dilemma: either she accepts that hypothetical explanatory knowledge may constitute an instance of genuine progress, and then she has to accept that Oresme's solution may have constituted progress, or she denies that hypothetical explanatory knowledge may constitute an instance of genuine progress, and then she has to accept that she is unable to assert that Einstein's work constituted progress in 1905. If she takes the first horn and accepts hypothetical explanatory knowledge, she has also to accept that an instance of progress may be considered as such independently of its confirmation. But, as we have seen above (sec. 2.2), Bird explicitly claims that whether a belief (or a theory, a solution, etc.) constitutes genuine knowledge has to be determined with respect to our current knowledge, i.e. from a point of view from which we can assess whether that belief has been confirmed or not. So, the supporter of the epistemic account has to take the second horn of the dilemma, and conclude that her account of progress is unable to assert that Einstein's work constituted progress in 1905 .

Thus, it seems fair to conclude that accepting hypothetical explanatory knowledge is not a viable proposal for the epistemic account, and that this account is unable to account for those cases that are equivalent to Einstein's case, i.e. cases in which an increase in the understanding of a phenomenon occurs, and we are justified in claiming that it in fact occurred, even if there was not a simultaneous increase in knowledge at the time it occurred.

It is worth underlining here that if, as Dellsén claims, the noetic account fares better than the epistemic account when dealing with this kind of case, this means that in Dellsén's view, whether a theory constitutes an increase in the understanding of a phenomenon at the time it is proposed, and so whether a theory constitutes progress, is independent of its later confirmation or rebuttal. In other words, the verdict on whether a theory provides an increase in the understanding of a phenomenon at time $t_{x}$, cannot be dependent on whether such theory will be confirmed and judged to be true in a specified later historical context $t_{x+n}$ (for example: "according to our best current knowledge", as in the case of Bird's evaluation of Oresme's solution), or in some unspecified later historical context (for example: "if the theory will be confirmed in the future", as in the case of hypothetical explanatory knowledge). The verdict on the increase of understanding (i.e. the capacity to explain or predict some aspect of the target phenomenon) has to be provided with respect to the context in which it occurred. 
PENULTIMATE DRAFT - PLEASE CITE THE PUBLISHED VERSION

To appear in: Danks, D.; Ippoliti, E. (eds.), Building Theories, Springer, Cham.

\subsection{Knowledge increase without progress}

Dellsén elaborates his proposal, and moves his criticisms to Bird's view, starting from a scenario which was proposed by Bird himself in his 2007 paper, and which was devoted to showing how an increase in scientific knowledge does not constitute progress in all circumstances. Bird describes this scenario as follows:

imagine a team of researchers engaged in the process of counting, measuring, and classifying geologically the billions of grains of sand on a beach between two points. Grant that this may add to scientific knowledge. But it does not add much to understanding. Correspondingly it adds little to scientific progress. (Bird 2007, p. 84).

It is easy to agree with Bird. This classificatory activity represents a genuine instance of accumulation of scientific knowledge, since the research produces true beliefs relative to the state of the world that are justified in a non-accidental way according to scientific standards and methods. And certainly, it is difficult to figure out how this activity may increase our comprehension of the world. Nevertheless, this apparently innocent consideration constitutes, according to Dellsén, an insurmountable difficulty for the supporters of the epistemic account, because they are unable to accommodate this consideration with the very qualifying claim of the epistemic account, i.e. that the trend of knowledge accumulation matches the trend of scientific progress. If this is the case, then for every instance of accumulated knowledge, however insignificant, we should identify a correspondent (i.e. equivalent) instance of scientific progress. If, on the contrary, an increase in scientific knowledge can occur without being correlated to an equivalent increase in progress, then we should deny that scientific progress has to be identified with knowledge accumulation, as the supporters of the epistemic account maintain.

It is interesting to note that Bird himself suggests that the discrepancy between knowledge accumulation and progress growth is due to whether understanding increases. Dellsén elaborates exactly on this suggestion to develop his noetic account, according to which scientific progress has to be identified with increase in understanding.

Examples equivalent to Bird's "counting grains of sand" scenario may be multiplied. Consider all those cases in which spurious correlations are identified and correctly deemed as such. ${ }^{7}$ Dellsén reports an interesting case:

it happens to be true that there is a strong correlation between increases in childbirth rates outside of Berlin city hospitals and increases in stork populations around the city. When this piece of information was published in $\mathrm{a}[\mathrm{n}]$ article that warned against coincidental statistical associations [...] there was an accumulation of knowledge that would have to count as scientific progress on the epistemic account. However, this information provides no understanding since it does not enable us to correctly explain or predict any aspect of childbirth rates or stork populations [...]. Intuitively, this is not a case of scientific progress. (Dellsén 2016a, p. 78).

7 On what spurious correlations are, cf. Dellsén 2016a, p. 78: "Suppose we have two variables $\mathrm{V}_{1}$ and $\mathrm{V}_{2}$ that are known on independent grounds to be unrelated, causally and nomologically. Let us further suppose that we learn, i.e. come to know, that there is some specific statistical correlation between $\mathrm{V}_{1}$ and $\mathrm{V}_{2}-\mathrm{e} . \mathrm{g}$. such that a greater value for $\mathrm{V}_{1}$ is correlated with a greater value for $\mathrm{V}_{2}$." This correlation represents an instance of spurious correlation, i.e. a correlation between two variables which is not due to any real relation between them. In these cases, such a correlation does not convey any information on the correlated variables, nor on some other relevant aspect of the world, so it is useless, irrelevant, or worse, it may lead us astray, if we do not identify it as spurious. 
To make Dellsén's remark more general, it may be useful to consider a paper recently published by Calude and Longo (2016). They base their argument, among other things, on Ramsey theory, i.e. the branch of combinatorics which investigates the conditions under which order must appear. If we restrict our attention to mathematical series, more precisely to arithmetic progressions, Ramsey theory investigates the conditions under which an arithmetic progression must appear in a string of numbers.

Calude's and Longo's analysis hinges on Van der Waerden's theorem, according to which for any "positive integers $k$ and $c$ there is a positive integer $\gamma$ such that every string, made out of $c$ digits or colours, of length more than $\gamma$ contains an arithmetic progression with $k$ occurrences of the same digit or colour, i.e. a monochromatic arithmetic progression of length $k$ " (Calude, Longo 2016, p. 11).

For example, if we take a binary string of $x$ digits, digits can be either ' 0 ' or ' 1 '. Take ' 0 ' and ' 1 ' to be the possible colours of those $x$ digits, i.e. $c=2$. From Ramsey theory, we know that there will be a number $\gamma$ such that, if $x$ is bigger than $\gamma$, that string will contain an arithmetic progression of length $k$ such that all $k$ digits of that progression are of the same colour, i.e. either all the $k$ digits are ' 0 ' or all the $k$ digits are ' 1 '.

Consider now a database $D$, where some kind of acquired information about some phenomenon $P$ is stored. We want to investigate the correlations among the data stored in $D$ in order to increase our knowledge of $P$ :

In full generality, we may consider that a correlation of variables in $D$ is a set $B$ of size $b$ whose sets of $n$ elements form the correlation [...]. In other words, when a correlation function [...] selects a set of $n$-sets, whose elements form a set of cardinality $b$, then they become correlated. Thus, the process of selection may be viewed as a colouring of the chosen set of $b$ elements with the same colour — out of $c$ possible ones. [...]. Then Ramsey theorem shows that, given any correlation function and any $b, n$ and $c$, there always exists a large enough number $\gamma$ such that any set $A$ of size greater than $\gamma$ contains a set $B$ of size $b$ whose subsets of $n$ elements are all correlated. (Calude, Longo 2016, p. 12). ${ }^{9}$

Calude and Longo prove that the larger $D$ is, the more spurious correlations will be found in it. In other words, when our stock of available data increases, most of the correlations that we can identify in it are spurious. Since large databases have to contain arbitrary correlations, owing to the size of data, not to the nature of data, the larger the databases are, the more the correlations in such databases are spurious. Thus, the more data we have, the more difficult is to extract meaningful knowledge from them. ${ }^{10}$

8 In this case (i.e. $c=2$ ), if we have $k=3$, then $\gamma=8$. To see this, consider the following sequence of binary digits of length 8: 01100110. This string contains no arithmetic progression of length 3 , because the positions $1,4,5,8$ (which are all ' 0 ') and 2, 3, 6, 7 (which are all ' 1 ') do not contain an arithmetic progression of length 3 . However, if we add just one bit more to that string (i.e. if we add either ' 1 ' or ' 0 '), we obtain the following two strings: 011001100 and 011001101. Both these strings contain a monochromatic arithmetic progression of length 3 . Consider 011001100: positions 1, 5, 9 are all ' 0 '. Consider 011001101: positions 3, 6, 9 are all '1'. More generally, it can be proved that if a string contains more than 8 digits, it will contain a monochromatic arithmetic progression of length 3 . And in fact, all the 512 possible binary strings of length 9 contain a monochromatic arithmetic progression of length 3 .

9 It is important to stress that the nature of the correlation function is irrelevant: it can be completely arbitrary, i.e. in no way related to the nature of the data stored in the database.

10 Cf. Calude, Longo 2016, p. 6: "it is exactly the size of the data that allows our result: the more data, the more arbitrary, meaningless and useless (for future action) correlations will be found in them." It may be interesting to note that, in order to derive their result, Calude and Longo define "spurious" in a more restrictive way than Dellsén. According to them, "a correlation is spurious if 
This result generalizes the difficulty deriving from the claim that scientific progress has to be identified with knowledge accumulation. Indeed, the search for spurious correlations within a stock of knowledge produces itself instances of knowledge, and so it contributes to the increase of the size of that stock of knowledge. But, as Calude and Longo demonstrate, the mere increasing of the stock of knowledge increases the number of spurious correlations that it is possible to find within it. Since, if our stock is sufficiently large, we can expect that most of the correlations that we can identify in it are spurious, we can also expect that the identification and the correct classification of these correlations as 'spurious correlations', while representing a genuine instance of knowledge, will hardly represent an instance of progress. So, the findings of spurious correlations are (almost) all cases in which knowledge increases while progress does not. And they are also cases that contribute to the increase of the number of possible spurious correlations, since they increase the stock of knowledge. Since the more spurious correlations are possible within a stock of knowledge, the more increasing our understanding becomes difficult, ${ }^{11}$ we may even hypothesize that, contrary to Bird's view, in certain circumstances knowledge accumulation may 'negatively' contribute to an increase in the understanding of relevant phenomena. ${ }^{12}$

According to Dellsén, the epistemic account is unable to account for all those relevant cases of knowledge production that do not imply an increase in progress, since it takes scientific progress to amount to knowledge accumulation. On the contrary, the noetic account is able to account for those cases, because while it acknowledges that those cases represent genuine instances of knowledge, it denies that they also constitute genuine instances of progress, given that they do not increase our understanding of the world, i.e. they do not enable us to correctly explain or predict any relevant aspect of the world.

\section{Knowledge and understanding: the problem of the factivity of knowledge}

We have seen that, according to Dellsén, the superiority of the noetic account with respect to the epistemic account is due to the fact that the noetic account is able to adequately account for some kinds of cases that the epistemic account is not able to adequately account for. In Dellsén's view, the inadequacy of the epistemic account in accounting for such cases is due to some qualifying features of the concept on which the epistemic account rests, i.e. "knowledge". In this perspective, the superiority of the noetic account is due to the fact that it rests on the concept of "understanding". In other words, it would be thanks to some features of the concept of understanding that the noetic account fares better than the epistemic account. The question then arises: What are the differences between the concept of "knowledge" and the concept of "understanding" that allow "understanding" to perform better than "knowledge" when we deal with scientific progress?

Before trying to answer this question, we have to keep in mind that we are dealing here with a realist perspective on science and scientific progress. The confrontation

it appears in a 'randomly' generated database" (p. 13). Details can be found in Calude, Longo (2016). In any case, this does not impinge on the considerations that follow.

11 Think ofthe increase in the understanding of some phenomenon $\mathrm{X}$ that may be derived by the findings of relevant (i.e. not-spurious) correlations among $\mathrm{X}$ and other phenomena $\mathrm{Y}$ and $\mathrm{Z}$ : if the number of spurious correlations increases, the number of correlations that we have to discard before finding the relevant ones increases too. Thus, increasing the understanding becomes more difficult when the number of spurious correlations increases.

12 On a similar point, see Rancourt (2015). 
between Bird and Dellsén takes place in a shared realist framework. Dellsén explicitly affirms that he considers his proposal to be a realist account of progress, ${ }^{13}$ and it is exactly for this reason that he takes the noetic account to be a valid alternative to Bird's epistemic account, which is a full-blooded realist view of progress. Dellsén sees the noetic account as a sort of amelioration of the epistemic account. In his view, the noetic account is a more adequate (and nuanced) realist account of progress than the epistemic one, since it is able to satisfyingly account for more kinds of cases.

Turning back to the differences between knowledge and understanding, one of the most salient is the strength of the truth and justification requirements: for a belief to be knowledge, it must be (at least) true and justified. On the contrary, in order to have some understanding of a phenomenon, what is required is the ability to make previsions or provide explanations relative to such phenomenon. ${ }^{14}$ But the history of science shows us that the capacity for providing explanations or making predictions may be due to beliefs or theories that are incomplete, (partially) false, or not yet empirically confirmed. So, the question is: Can this ability of making predictions and providing explanations, i.e. understanding, really be decoupled from the truth requirement?

There is a bifurcation here: indeed, it is very possible to give a realist interpretation of the concept of understanding, according to which the ability to make predictions and provide explanations depends, in the ultimate analysis, on the truth of the theory we are dealing with; but another interpretation of the concept of understanding is also possible, which, since it is opposed to the previous one, can be labeled "anti-realist", according to which the abilities of making predictions and providing explanations are not necessarily related to the truth of the theory we are dealing with. In other words, according to the realists, scientific progress cannot be due to anything other than an approximately true theory, while according to the anti-realists even a false theory may constitute a genuine scientific progress..$^{15}$ Thus, a realist interpretation of the concept of understanding gives rise to a realist account of scientific progress, while an anti-realist interpretation of the concept of understanding gives rise to an anti-realist account of scientific progress (see e.g. Khalifa 2011; de Regt, Gijsbers 2017). Both those interpretations have been advanced and defended in the last years. ${ }^{16}$

Consider now that we are dealing here with the noetic account, and that, as we have already noted above, Dellsén takes it to be a realist account of progress. So, we can conclude that Dellsén adopts a realist stance on understanding, and thinks that, in the

13 Cf. Dellsén 2016, p. 73, fn. 6: "the noetic account amounts to a moderately realist view of the aim of science."

14 Several very different views have been advanced on the requirements that have to be fulfilled in order to have understanding (see for a survey Baumberger, Beisbart, Brun 2017). The main distinction is between those authors who think that understanding is just a species of knowledge (and so there is not a real distinction between these two concepts, see Grimm 2006), and those who, on the contrary, think that understanding is not just a species of knowledge (see Dellsén 2016b). Those who belong to this latter group have different ideas on how exactly understanding differs from knowledge. They usually claim that understanding lacks one or more of the traditional knowledge requirements, i.e. truth, justification, and some anti-luck condition. Here we will follow Dellsén's characterization of understanding, and assume, at least for the sake of the argument, that understanding is not just a species of knowledge.

15 The idea that empirical success is a good indicator of truth is a pillar of scientific realism (see e.g. Wray 2013). Thus, a realist view of scientific progress cannot completely sever the link between the empirical success of a theory and its truth.

16 See e.g. Kvanvig (2003), who maintains that the truth requirement is necessary for understanding; Elgin (2007), who maintains that we may have understanding even through falsities; Rancourt (2015), who maintains that, in certain circumstances, an increase in truthcontent may even lead to a decrease in understanding. 
ultimate analysis, understanding depends on the truth of the theory we are dealing with. But if understanding depends on truth as knowledge does, how is it possible that it differs from knowledge in such a way that the noetic account is able to account for those cases that the epistemic account is not able to account for precisely because of the rigidity of its truth requirement?

Dellsén, in order to maintain both the claim that there is a relevant difference between understanding and knowledge with respect to the truth requirement, and the claim that the noetic account, which is based on the concept of understanding, is a realist account of progress, adopts a line of reasoning developed by some epistemologists in recent years, and takes understanding to be a quasi-factive concept. ${ }^{17}$

Knowledge is usually considered to be factive: if a person has the belief that $p$, she does not know that $p$ unless ' $p$ ' is true. ${ }^{18}$ Understanding would instead be quasi-factive, in the sense that it is not necessary that each component of a theory be true to make this theory able to increase understanding. It is sufficient that "the explanatorily/predictively essential elements of a theory" be "true in order for the theory to provide grounds for understanding" (Dellsén 2016a, p. 73, fn. 6). On this view, at the "periphery" of a theory there well may be inaccuracies, falsities, abstractions, idealizations, i.e. all those elements that prevent us from baldly claiming that a theory is straightforwardly "true" and that everything it states constitutes "knowledge". And those elements may even contribute in some way and to some extent to the understanding of the target phenomenon. But what, in ultimate analysis, grounds our understanding of a phenomenon is the truth of the "central propositions" of the theory we are dealing with (Kvanvig 2009; Mizrahi 2012).

In this way, the abilities of making predictions and providing explanations of some phenomenon are not completely severed by the possession of some relevant truths about this phenomenon. And according to Dellsén, this would be sufficient both (1) to consider the noetic account a realist account of progress, and (2) to consider genuine contributions to progress those cases in which understanding increases thanks to a theory replete with elements that are not strictly true, i.e. elements whose presence prevents us to claim that knowledge also increases, since knowledge is a more demanding concept.

If we conceive of understanding in this way, understanding admits of degrees, while knowledge is an all or nothing matter. There is no such thing as degrees of knowing: either you know or you don't. This is mainly due to the fact that truth is an all or nothing matter. Since knowledge requires truth, and truth does not admit of degrees, knowledge does not admit of degrees in its turn. Thus, understanding may increase even if there is not an increase in the truth content of the theory we are dealing with, if some, strictly speaking, false elements of a theory allow us to make better predictions or provide better explanations of the target phenomenon. From this, it follows that while it makes no sense to claim that a true theory is more true than another true theory, it makes perfect sense to claim that a theory provides a better understanding of a phenomenon than another theory. Since there may be an increase in understanding even if there is not an increase in the truth content of a theory, there may be an increase in the understanding of a phenomenon even if there is not an increase in our knowledge relative to such phenomenon.

17 On the quasi-factivity of understanding, see Kvanvig (2009), and Mizrahi (2012). For some criticisms of this view, see Elgin (2009), and de Regt, Gijsbers (2017).

18 Many authors argue that knowledge is a factive propositional attitude. To say that a propositional attitude is factive is to say that "it is impossible for you to have that attitude towards anything other than a true proposition" (Pritchard, Turri 2014), where "true" has to be intended in the sense of "corresponding to facts". Williamson (2000) argues that knowledge is the most general factive propositional attitude. For a radically different view on knowledge, see Cellucci (2017). 
According to Dellsén, those features of the concept of understanding make it perfectly suited to account for scientific progress, and for other relevant aspects of scientific practice, such as the use of highly idealized models in science.

\section{The noetic conception as a realist conception of progress}

In order to assess Dellsén's claim that the noetic account is a more adequate realist account of progress than the epistemic account, we will leave aside many of the criticisms that have been raised against the quasi-factive view of understanding. ${ }^{19} \mathrm{We}$ will instead try to develop an argument elaborating on some works of Barrett (2003, 2008), Stanford (2003), and Saatsi (2016). This argument aims at showing that, if understanding is quasifactive, the noetic account may face some difficulties in accounting for the case of the incompatibility of Quantum Mechanics (QM) and General Theory of Relativity (GTR), and that this impinges on Dellsén's claim that the noetic account is an adequate realist view of progress.

QM and GTR are certainly our current best scientific theories, in any sense in which 'best' is usually intended by philosophers of science. In a realist perspective, since scientific realism claims that our best theories are true (Chakravartty 2015), both QM and GTR should be regarded as true or, at least, approximately true. Thus, a realist view of scientific progress should account for QM and GTR accordingly.

But there is a problem. Roughly, we know that QM and GTR are incompatible, in the sense that, due to the different fundamental theoretical assumptions they make, they provide two contradictory descriptions of the world (see e.g. Macías, Camacho 2008). And to the extent that two incompatible theories cannot both be true, we know that QM and GTR cannot both be true (Barrett 2003; Bueno 2017). ${ }^{20}$

Let's try to consider how Dellsén's proposal would account for this case. Since the noetic account rests on the concept of understanding, and conceives of understanding as our ability in making predictions or providing explanations, if we adopt the noetic view, we should admit that QM and GTR constitute genuine cases of scientific progress. Indeed, since they are the most powerful theories we have, we can fairly affirm that they allow us to make accurate predictions and provide deep explanations of a large class of phenomena.

But recall that according to Dellsén, the noetic account rests on a quasi-factive conception of understanding. In this view, the abilities of making predictions and providing explanations that QM and GTR give us, have to be due, in ultimate analysis, to the truth of the "essential elements" of those theories. If this is the case, then QM and GTR may be deemed to be (approximately) true, at least in their essential theoretical 'core'. But if QM and GTR are both (approximately) true (at least) in their essential theoretical 'core', they should not be radically incompatible, at least in principle. Nevertheless, there is a wide consensus among scientists and philosophers of science on the claim that QM and GTR are in fact radically incompatible.

19 The main criticism to the quasi-factive view of understanding is the one developed by Elgin (2007; 2009), who shows how in many cases idealizations and other 'untrue' elements of a scientific theory are "central terms" of that theory, not peripheral. They are "essential elements of a theory" that cannot be replaced, nor are expected to be replaced in the future by scientists.

20 It is worth noticing that this argument does not rest on a sort of pessimistic induction over past science (Laudan 1981), as many anti-realist arguments do. And so it does not display the weakness that is usually thought to afflict that kind of arguments, i.e. their inductive character (Barrett 2003). Indeed, the argument we are dealing with here is based on the theoretical impossibility of reconciling the images of the world provided by our current most powerful scientific theories. 
There seems to be a tension here. As we will see below, the supporter of the noetic account may try to solve this difficulty either by accepting the claim that QM and GTR are incompatible, and so that one of those theories is false (sec. 5.1), or by denying this claim, and so maintaining that both those theories are true (sec. 5.2).

\subsection{First option: affirming the incompatibility of QM and GTR}

Let's consider the hypothesis that QM and GTR are really incompatible, and so they cannot both be true. This would mean that even the 'essential elements' of at least one of QM and GTR are not true. Since, as we have seen, the supporter of the noetic account should consider both QM and GTR to be cases of progress, this leads her to a dilemma: 1) either she has to admit that the noetic view is not able to account for the fact that a theory whose 'essential elements' are false may nevertheless increase our understanding; 2) or she has to claim that such a theory has never really increased our understanding, given that its 'essential elements' were false.

Both the horns of this dilemma are problematic to take. Indeed, if one takes 1), one has to admit that the noetic account is an inadequate account of scientific progress, because it is not able to account for such a relevant case, i.e. that a radically false theory may constitute progress. If, on the other hand, the supporter of the noetic account takes 2 ), she finds herself in a position that is in some way similar to the one in which the supporter of the epistemic account finds herself if she tries to consider Einstein's work a case of progress (see above, sec. 3.1).

Let's try to see why. As we have seen, according to Dellsén's criteria, both QM and GTR are cases of progress, since they increase our understanding. But according to the quasi-factive view of understanding, if QM and GTR are radically incompatible, they cannot both be genuine cases of progress. If we want to maintain the quasi-factive view, we have to deny that one of QM and GTR really provides understanding. But at the moment we are not able to assess, between QM and GTR, which is the approximately true theory and which is the one that will be discarded because its essential elements will be proven false. According to our current standards, they are both extremely empirically successful.

To face this difficulty, we may be tempted to claim that QM and GTR provide us just a sort of hypothetical understanding, i.e. the understanding that a theory would provide if its essential elements are true. In this view, when in the future the theory whose essential elements are true will be identified, we will be able to assess which theory between QM and GTR really increases our understanding, and so constitutes a genuine case of progress. But if the verdict on the progressiveness of QM and GTR is made dependent on (an eventual) future confirmation of the truth of their essential elements, we would not be really able to claim now that they constituted genuine cases of progress when they were formulated, exactly as the epistemic account was unable to claim that Einstein's paper constituted a genuine progress when it appeared in 1905.

But the idea of making our judgment over the progressiveness of a case dependent on future confirmation is exactly what Dellsén deemed unacceptable in considering the attempt of rescuing the epistemic account with respect to Einstein's work by considering it a case of hypothetical explanatory knowledge. Now, as we have seen, the epistemic account faces a dilemma: either it is not able to consider Einstein's work a case of progress, or, if it tries to rely on the idea of hypothetical explanatory knowledge to account for such a case, it is no more able to rule out Oresme's solution from the set of genuine cases of progress. In a similar way, the noetic account faces a dilemma with regard to QM and GTR: either it is not able to rule out the false theory between QM and 
GTR, or if it tries to rely on the idea of hypothetical understanding to discriminate between these two cases, it is no more able to claim now whether they are genuine cases of progress or not.

Moreover, the strategy of making our judgment over the progressiveness of a case dependent on future confirmation faces two more general problems. The first is the risk of a regress. If at any time $t_{x}$, the supporter of the noetic account has to wait for the truth of the essential elements of our current best theory to be confirmed by future science at time $t_{x+n}$ in order to judge its progressiveness, then she risks being able to correctly determine what episodes in the history of science constituted genuine cases of progress only at the end of time. Indeed, the truth of the essential elements of any past theory can be determined only in the light of our best current theory. But the truth of the essential elements of our best current theory is determinable only by future science (Barrett 2008; Stanford 2003; more on this below). It is also conceivable that in the light of future science, say at time $t_{x+n}$, the essential elements of some past theory $T$ that we now, at time $t_{x}$, deem true in the light of our current science will be discarded and regarded as false. So, there is the risk that we may have to modify our verdicts on the progressiveness of any past episode at any future time.

The second general problem that this strategy faces is that, if we have to wait for some future confirmation to judge over the progressiveness of an episode, the distinction between the noetic account and the epistemic account fades. Indeed, Dellsén explicitly argues (see above, sec. 3.1) that these accounts are distinct because the noetic account is able to determine whether a theory is progressive by focusing on the historical context in which it is proposed, i.e. on the contribution to the understanding of a phenomenon that it provides at the time of its formulation, independently of whether this theory will later be confirmed or not, while the epistemic account, since it rests on the concept of knowledge, has to wait for a theory to be confirmed to regard it as an instance of progress. If confirmation is also required by the noetic account, the noetic account becomes equivalent to the epistemic account. ${ }^{21}$

To conclude, if we take 2), i.e. we claim that one of QM and GTR has never really increased our understanding, since its 'essential elements' are false, this would amount to admit that there may be cases in which an increase in our ability to make predictions and provide explanations increases while our understanding does not increase. This kind of case could not be accounted for by the noetic view, since these cases contradict the qualifying claim of the noetic account, namely that scientific progress has to be identified with an increase in understanding.

Thus, since neither 1), nor 2) seem to be a viable route to take for the supporter of the noetic account, it is fair to conclude that the first option we considered, i.e. affirming that QM and GTR are really incompatible, is not a promising way to solve the problem of accounting for the incompatibility of QM and GTR if we adopt the noetic account.

21 There are some additional difficulties for Dellsén's account worth being pointed out: the first is that it is true that if QM and GTR are incompatible, at least one of them cannot be true. But we cannot exclude that neither is. How would the noetic view account for the possibility that, in light of future science, the essential elements of both QM and GTR will be deemed untrue? Shall we then claim that neither QM nor GTR were cases of progress when they were formulated and applied? In the same vein: even if we concede that future confirmation will allow us to determine that one theory between QM and GTR really increases our understanding because of the truth of its essential elements, there will still be the difficulty for the supporter of the noetic account to explain the empirical success enjoyed by the other radically 'false' theory, given that it is uncontroversial that both QM and GTR are extremely successful in dealing with the world, and that the noetic account claims to be a realist account of progress. 


\subsection{Second option: denying the incompatibility of $Q M$ and GTR}

The supporter of the noetic account may try to defend her view by claiming that QM and GTR are not really incompatible, that they are both approximately true theories, and that their now seeming incompatibility derives exclusively from some of their non-essential elements, which could be amended in the future. If this is the case, both QM and GTR can be regarded as genuine cases of progress in light of the noetic account.

There are two main and related problems with this line of reasoning. The first is that the claim that QM and GTR are not really incompatible contradicts the 'received view' among scientists and philosophers on this issue. Obviously, this is not a decisive rejoinder, but it is a reminder of the fact that the burden of proof of the claim that such a deep incompatibility as the one that obtains between QM and GTR may be due to the incompatibility of just some peripheral elements of those theories, is on the supporter of this line of defense. And proving such a claim is not an easy task (Barrett 2008).

The second, and more difficult, problem is that this defense of the noetic account postpones the ascertainment of the (approximate) truth of QM and GTR to some future time, and this makes this line of defense ineffective.

Indeed, QM and GTR are our current best theories. From a realist point of view, we can at most hypothesize that they are (approximately) true, since they are actually enjoying huge empirical success. Nevertheless, as realists themselves usually acknowledge, this kind of inference "from the success to the truth" of a theory is insufficient to establish with certainty that a theory is actually true. There is always the possibility that a theory, even if by our current standards empirically successful, will be dismissed and deemed to be false in light of future science. Thus, we cannot, at the moment, estimate the degree of approximation to the truth of QM and GTR, nor can we, strictly speaking, even really claim that they are "approximately" true in any meaningful sense, since we cannot assess their approximation to the truth in the same way we usually do in order to maintain that a past theory is approximately true. Indeed, we can claim that a past theory $T$ was approximately true, only by comparing it with our best (and more complete) current theory $T^{*}$ of the same domain (Stanford 2003). In the case of QM and GTR, since they are our current best theories, we cannot estimate their approximation to the truth by comparing them to a more complete theory that we deem to be true (Barrett 2008).

To better see this point, consider Newtonian Gravitation (NG): realists may claim that NG is approximately true, since they are able to compare NG to GTR, and thus show that the former can be derived as a special case of the latter (Saatsi 2016; Barrett 2008). But it would be pointless to claim that GTR is "approximately true", since we do not know what it may approximate. Since GTR is so successful, we use it to estimate the degree of "approximation" to the truth of past theories, but we cannot say that GTR is approximating some more true theory. Nor can we claim that GTR is the true and definite theory, since we know that it is theoretically unable to explain the phenomena pertaining to a relevant domain of nature (the domain of QM), and that it is incompatible with QM. So, even if we think that QM and GTR are not really radically incompatible, and that they are both approximately true, this very fact means that none of them can be the definite theory. At most, it may be claimed that they both approximate the same future definite theory.

It may be claimed that in the future there will certainly be developed a theory TX, and that it will be possible to show that GTR approximates TX, in the same way in which it has been possible to show that NG approximates GTR. But this claim is just as compelling as any other induction is, since it rests on our hope that the future will 
resemble the past. We have no effective way to support the claim that GTR will be proven to approximate TX, since we have no way to know what TX may look like. Since we are unable to compare TX and GTR, we are unable to assess whether GTR approximates TX. We cannot rule out the possibility that GTR and TX will be radically distinct, and that the essential elements of GTR will be deemed to be false in the light of TX. If this is the case, GTR would be a false but empirically successful theory, and so it cannot be claimed to be approximately true. Given that QM and GTR are incompatible, and that this may imply that GTR is in reality radically false (since, even if we are now considering the possibility that QM and GTR are not radically incompatible, we have not yet succeeded in showing that in fact they are compatible), this scenario is not implausible, and so cannot be easily dismissed. This argument may be developed, mutatis mutandis, with regard to the claim that $\mathrm{QM}$ will approximate TX.

It may be objected that if QM and GTR are not really incompatible, i.e. their incompatibility is due just to some of their peripheral elements, then it is not true that TX is completely undetermined. We have some clues of how TX should be: if we take that GTR's essential elements are true, since GTR constitutes progress and we adopt a realist view of progress, then TX should retain GTR's essential elements and dismiss just the false, peripheral elements of GTR. Moreover, because of this continuity between GTR and TX, TX should be such that GTR may be derived from it as a special case, in the same way in which NG is derivable from GTR.

The problem is that, even if we concede that TX will have to meet these constrains, we are nevertheless still unable to effectively determine whether GTR really approximates TX. The fact is that we have no idea how GTR should be modified in order to be improved. ${ }^{22}$ We do not know now what elements will be deemed central and what elements will be deemed peripheral, and so dismissed, in the future in light of TX (Barrett 2003). ${ }^{23}$ Such a distinction between central and peripheral elements will be possible only after TX will have been elaborated and confirmed. Think again of NG. If we accept the line of reasoning we are analyzing, we should maintain that, by reasoning alone, at Newton's time, it would have been possible to determine (at least to some extent) the form that GTR should have taken, and what elements of NG would have consequently been deemed essentials. If this were the case, it would have been possible to compare NG and GTR (before this last one had even been actually elaborated), and safely claim that NG approximates GTR. But things go the other way around. It is starting from GTR that we can now claim what elements of NG are essential and what "errors" or "falsities" are embedded in NG. And it is in large part due to the post-GTR development of a geometrized version of NG, which is empirically equivalent to NG, "but absorbs all the information about gravitational forces into information about spacetime curvature so that gravitation is rendered a manifestation of spacetime curvature" (Saatsi 2016, p. 9), that we are now able to understand how NG may be shown to be a special case of GTR, and thus why we can safely claim that NG approximates GTR. ${ }^{24}$

22 Cf. Barrett 2003, p. 1216: "While we do have a vague commitment that our future physical theories will somehow be better than our current physical theories, we do not now know how they will be better. If we did, we would immediately incorporate this insight into our current physical theories."

23 Cf. Barrett 2003, p. 1216: "insofar as we expect surprising innovations in the construction of future theories [...], we cannot now know even what the structure of the space of possible options for refining our current theories will prove to be." This point cannot be developed here, but it is worth underlining that this line of reasoning is analogous to the unconceived alternatives argument developed by Stanford (2006), who elaborates on Sklar (1981).

24 See also Barrett (2008). For more details, see Malament (1986a, 1986b). 
To sum up, if we try to defend the noetic account maintaining that QM and GTR are not really incompatible and are both approximately true, in order to make our defense effective, we should be able to compare QM and GTR to the theories (or the theory) that will replace them in the future. But, since we have no idea about what such future theories may look like, we are not able to show that QM and GTR approximate them, and so we cannot claim that QM and GTR are approximately true. So, it does not seem easy to find a way to defend the claim that the incompatibility between QM and GTR is due just to some of their peripheral elements. Thus, taking the second option, i.e. maintaining that QM and GTR are not really incompatible, does not seem to be a promising way to defend the noetic account.

Since neither the first option nor the second option we considered seems to provide a viable route to take for the supporter of the noetic account, we can fairly conclude that the problem of the incompatibility between QM and GTR represents a serious challenge for the noetic account.

\section{Factivity and modality}

From what we have said so far, it seems that the supporter of the noetic account faces a dilemma: either 1) she dismisses the noetic account as inadequate, or 2) she dismisses her quasi-factive view of understanding. Indeed, if she tries to rescue the realist construal of the noetic account by adopting a full-blooded factive view of understanding, she risks being unable to distinguish understanding from knowledge, and so to let the noetic account become equivalent to the epistemic view. But if she discards the quasi-factive view and adopt a non-factive view of understanding, she has to dismiss the claim that the noetic account is a realist account of progress, since adopting a non-factive view would amount to admitting that even a radically false theory may constitute progress, a claim that realists usually deny.

But there may still be another option for the supporter of the noetic account who wants to maintain the claim that the noetic account is a realist account of progress. Rice (2016) has recently claimed that we can construe the idealized models commonly used in science in a realist fashion, even if we concede that they do not accurately represent the way the world really is, and that they indispensably rest on idealized assumptions, i.e. even if some of their essential elements are known to be false. Rice's aim is to solve the difficulty that the realist has to face when she tries to account for the role that idealizations play in science, a theme deeply connected with the debate on the concept of understanding (Elgin 2009; Saatsi 2016). In a nutshell, Rice suggests that highly idealized models may nevertheless provide factive scientific understanding, since they give us true modal information about the counterfactual relevance and irrelevance of various features of the target system. So, despite their representations being inaccurate and many of their assumptions being false, those models may increase our understanding. This view of understanding seems to be compatible with the noetic account, because, despite the fact that Rice names his view of understanding 'factive', he does not construe 'factive' in a too demanding way as necessarily requiring truth: "my view is that scientific understanding is factive because in order to genuinely understand a natural phenomenon most of what one believes about that phenomenon [...] must be true" (Rice 2016, p. 86). This may be sufficient for distinguishing the concept of 'understanding' from the concept 
of 'knowledge'. Thus, it seems, at least prima facie, that Rice's proposal may be compatible with Dellsén's account of progress. ${ }^{25}$

Relying on Rice's proposal, the supporter of the noetic account may try to account in a realist fashion for those cases in which a theory provides an increase in our understanding even if its essential elements have to be considered, strictly speaking, false. This kind of case is exactly the one we analysed above (sec. 5) with respect to the incompatibility between QM and GTR. By adopting Rice's view, the supporter of the noetic account may claim that, even if in the future one of QM and GTR (or both of them) will be deemed to be strictly speaking false, this false theory is nevertheless really increasing our understanding, and so it constitutes a genuine instance of progress. She may also maintain that the previous claim is compatible with the idea that the noetic account is a realist conception of scientific progress, arguing that such a false theory, despite the falsity of its essential elements, provides us true modal information on some relevant aspect of the pertinent phenomena, and it is this true modal information that is responsible for the increase of our understanding. Since in this view the truth requirement is, at least to some extent, fulfilled by the modal essential element of the theory, this may well be claimed to be a realist view of progress: "it is the model's ability to provide true modal information about the space of possibilities that enables the model to produce" scientific understanding (Rice 2016, p. 92).

The main problem with this approach is that, in order to salvage the claim that only true elements may be responsible for an increase in understanding, it assumes that a false theory may convey true modal knowledge.

Now, let's concede, for the sake of the argument, that it may be possible to provide a coherent realist notion of "a false theory that is able to convey true modal information", and that it may also be possible to provide an account of how a theory such as QM or GTR may be considered an instance of this kind of false theory. The problem on which we will focus is that this 'move' amounts to embracing modal realism. In other words, if the supporter of the realist account tries to salvage her realist view of progress by relying on Rice's proposal, she commits herself to modal realism. But this commitment may be challenging for her.

Indeed, if a theory is supposed to be able to provide true modal knowledge, this means that we assume that modal knowledge is possible, i.e. that we are able to know what is necessary and what is possible. Since we are dealing here with a realist conception of understanding, and since the theory we are considering is supposed to be false, the element that has to be true, in order to fulfill the realist truth requirement, is the modal information provided by the theory. Indeed, if we take an anti-realist stance on modality, nothing is left in the theory which can be regarded as 'true' from a realist point of view, and so Rice's conception of understanding cannot be applied, nor can the noetic account be claimed to be a realist account of progress anymore. So, if we adopt Rice's proposal to support the noetic account, we have to take the modal information provided by the theory as true, and we have to intend 'true' in a realist sense. In the context of modal epistemology, this usually amounts to adopting some formulation of 'possible worlds' modal realism, a view which has been fiercely disputed and it is certainly not easy to defend (Vaidya 2016; Bueno 2017).

It may be objected that the true modal information provided by the false theory refers only to some actual features of the relevant phenomena, and that this fact disposes us to

25 Some work would be necessary to generalize Rice's proposal and make it suitable to account for the case of the incompatibility between QM and GTR, since Rice (2016) refers just to some kinds of models, and especially focuses on some optimality models used in biology, while QM and GTR are theories. This point cannot be developed here, but a promising route may be to adopt the semantic view of theories, according to which a theory is the class of its models. 
commit ourselves to some possible-worlds construal of modality, since in this case we do not have to account for non-actual possible features of the relevant phenomena, and so we can avoid making reference to non-actual possibilities in terms of possible worlds, which are ontologically dubious and epistemically inaccessible entities. Indeed, if the theory tells us what is actual, from what is actual we can safely infer what is actually possible without making reference to any world other than ours (Bueno 2017).

But, even granting for the moment the 'soundness' of this objection, it seems to be inadequate. Let's try to see why. Rice (2016) distinguishes between system-specific models and hypothetical models. System-specific models provide accurate information about the counterfactual relevance and irrelevance of salient features of the target system. On the contrary, hypothetical models are not intended to accurately represent any particular features of a real-world system, i.e. "the model has no real-world "target system' whose (difference-making) features it aims to accurately represent" (Rice 2016, p. 91). To sum up, "system-specific models aim to provide 'how actually' information while hypothetical models typically aim to provide 'how possibly' information" (Rice 2016, p. 92). Now, if we try to adapt Rice's proposal to the noetic perspective on the falsity of one of QM and GTR, we have to keep in mind that here we are dealing with a theory that is, by hypothesis, radically false, i.e. whose essential elements are false. So, it is reasonable to think that such a theory would be considered, in the modified noetic account that we have assumed it is possible to elaborate according to Rice's proposal, as being analogous to Rice's hypothetical models, and not to Rice's system-specific models. Indeed, if such a theory is radically false, this means that it does not accurately tell us anything about the actual target system, otherwise some of its essential elements would have been true. If this is the case, the objection mentioned above does not hold, since hypothetical models do not provide us with modal information about actual features of the target phenomenon; they instead provide information about non-actual possibilities of the target phenomenon: they "explore the possibility space in which features differ [...] dramatically from those of the actual system" (Rice 2016, p. 92, fn. 12). Thus, this is not a viable route for the supporter of the noetic account to avoid the commitment to some formulation of possible-worlds modal realism.

\section{Understanding and modalism}

There are several difficulties that arise for the supporter of the noetic account from her commitment to such a disputed view as possible-worlds modal realism. ${ }^{26}$ Perhaps the main difficulty comes from the fact that the noetic account aims to be a realist account of scientific progress. Many scientific realists think of themselves as naturalists, i.e. they adopt a naturalist stance (Morganti 2016). But it is at least very controversial whether possible-worlds modal realism may be compatible with a naturalist stance. Indeed, modal realists claim that, for every way the world could be, there is a world that is that way (Lewis 1986). This means to assume that if something is impossible in our world but it is conceivable, it is true in some other possible world causally isolated from ours. This contrasts with the way 'naturalism' is usually construed, i.e. as implying that natural entities are all there is, and that for an entity to be regarded as natural, it has to not be in principle spatiotemporally and causally isolated from our world (Papineau 2016). ${ }^{27}$

26 For a survey of the problems afflicting possible-worlds modal realism, see Vaidya (2016), Bueno (2017), Bueno, Shalkowski (2004).

27 The adoption of possible-worlds modal realism amounts to assuming that there is something "like a realm of metaphysical possibility and necessity that outstrips the possibility and necessity 
In any case, we will put aside these difficulties here, and we will grant that there is an escape route for the supporter of the noetic account, i.e. another promising realist view of modality to take, which does not rest on possible worlds, i.e. modalism, according to which modality is a primitive notion (Bueno 2017; Bueno, Shalkowski 2004).

Let's grant, for argument's sake at least, that modalism is able to avoid or solve many of the problems that afflict possible-worlds modal realism (Vaidya 2016, sec. 1.2.3). The problem that we will try to point out is that this account of modality seems to be in conflict with a (quasi-)factive view of understanding, i.e. the view of understanding advocated by Dellsén.

Modalism draws on an analogy between modal and mathematical knowledge originally developed by Lewis (1986), but it reaches different conclusions:

the key idea is that we have mathematical knowledge by drawing (truth-preserving) consequences from (true) mathematical principles. And we have modal knowledge by drawing (truth-preserving) consequences from (true) modal principles. (Bueno, Shalkowski 2004, p. 97).

According to this view, to know that $P$ is possible (or necessary) means to derive "it is possible that $P$ " from particular assumptions. More precisely, to know that $P$ is possible amounts to being entitled to introduce a possibility operator: it is possible that $P$. In some cases, it is easy to do so: we know that $P$ is actual, and therefore possible. But in many cases, $P$ is not actual. So, in these cases, when we claim that 'we know that $P$ is possible', what we are really doing is deriving it is possible that $P$ from some particular assumptions.

Here the different way in which Bueno and Shalkowski interpret the analogy between modality and mathematics marks their distance from Lewis's approach. In a nutshell, they develop this analogy as implying that in those cases in which we cannot know (by empirical means) that $P$ is actual, the modal knowledge that we can at most reach is conditional, i.e. it is of the form: " it is possible that $P$ ' is true, given that the assumptions on which we rest to derive 'it is possible that $P$ ' are true».

As in mathematics, due to Gödel's results, we are generally unable to prove with certainty that the axioms of the theory we use to derive a theorem $T$ are 'true', and we take our knowledge of such theorem to be of the form: «the theorem $T$ is true, if the axioms from which it is derived are true»; when dealing with modality we are unable to prove that the modal principles that we choose in order to derive the target conclusion are true. ${ }^{28}$ Indeed, in many cases the possibility of determining whether something is possible

that science deals with, but this is exactly what naturalists should not be willing to concede" (Morganti 2016, p. 87).

28 On the consequences of Gödel's results for how mathematical knowledge should be conceived, see Cellucci $(2017,2013)$. On how modalism construes the analogy between modality and mathematics, cf. Bueno, Shalkowski 2004, p. 97-98: "If the analogy with mathematics is taken seriously, it may actually provide a reason to doubt that we have any knowledge of modality. One of the main challenges for platonism about mathematics comes from the epistemological front, given that we have no access to mathematical entities - and so it's difficult to explain the reliability of our mathematical beliefs. The same difficulty emerges for modal realism, of course. After all, despite the fact that, on Lewis' account, possible worlds are concrete objects, rather than abstract ones, we have no access to them. Reasons to be skeptical about a priori knowledge regarding mathematics can be easily 'transferred' to the modal case, in the sense that difficulties we may have to establish a given mathematical statement may have a counterpart in establishing certain modal claims. For example, how can we know that a mathematical theory, say ZFC, is consistent? Well, we can't know that in general; we have, at best, relative consistency proofs. And the consistency of the set theories in which such proofs are carried out is far more controversial 
or not will depend on controversial assumptions. There are several incompatible and competing assumptions available to be taken as the starting point from which we derive our target conclusions on what is possible, and there is not a way of proving that such 'first assumptions' are at their turn 'true' without ending in an infinite regress or committing a petitio principii.

According to modalism, we have to accept that with respect to cases involving nonactual possibilities, "instead of having categorical modal knowledge [...] (that is, knowledge of what is possible or necessary, independently of particular assumptions)," we can at most "have conditional modal knowledge [...] (that is, knowledge of what is possible or necessary given certain philosophical assumptions)" (Bueno 2017, p. 80).

Now, in the context we are dealing with, as we have already seen above, the radically false theory between QM and GTR, which is supposed to be able to give us true modal knowledge, is supposed to convey modal information relative to non-actual possibilities. Thus, if we adopt modalism to escape the conundrums deriving from possible-worlds modal realism, we have to consider the modal knowledge provided by this false theory an instance of conditional knowledge. The problem is that conditional knowledge is unable to fulfill the realist requirement that is necessary to claim that the noetic account is a realist account of scientific progress. Indeed, if we adopt Dellsén's and Rice's (quasi)factive view of understanding, instances of conditional modal knowledge cannot be considered to be able to increase our understanding, since we cannot assess whether they are really true.

Thus, modalism is not really a viable route to take for the supporter of the noetic account: if she tries to rely on Rice's proposal and 'go modal' to face the challenge deriving from the incompatibility of QM and GTR, she seems unable to avoid committing herself to possible-worlds modal realism.

\section{Conclusion}

In this article, we have tried to assess whether the noetic account is a more adequate realist account of progress than the epistemic account. We elaborated an argument that aims to show how the quasi-factive view of understanding that Dellsén adopts in order to maintain that the noetic view is a realist account of progress is in tension with Dellsén's definition of understanding. We examined a possible way out for Dellsén, the adoption of Rice's proposal that highly idealized models may nevertheless provide us factive scientific understanding by giving us true modal information about pertinent phenomena. But this shift to modality comes with a cost for the supporter of the noetic account: it implies that she has to commit herself to possible-worlds modal realism, an option that may be unpalatable for many scientific realists. Finally, we have proposed another way out for Dellsén, i.e. the adoption of modalism. But we showed that modalism is not able to support a realist view of understanding.

To sum up: if the supporter of the noetic account wants to maintain the standard way to conceive of knowledge and understanding, she faces the following dilemma: either 1) she dismisses the noetic account as an inadequate realist account, or 2) she dismisses her quasi-factive view of understanding. If she tries to escape this dilemma by 'going modal', she faces a new dilemma: either 1) she commits herself to possible-worlds modal realism, or 2) she dismisses her quasi-factive view of understanding.

than the consistency of ZCF itself, given that such theories need to postulate the existence of inaccessible cardinals and other objects of this sort." 
In both these dilemmas, to take option 1) is very difficult: in the first case, it amounts to admitting that the noetic account does not really fare better than Bird's view, while in the second case, it implies adopting such a controversial perspective on modality that many scientific realists may tend to prefer Bird's view in any case. If one of the main rationales for develop the noetic account was advancing the epistemic view, this seems not to be a good result. We think that option 2) is the more promising one. Obviously, taking 2) amounts to dismissing the idea that the noetic account is a realist account of progress, and this may seem an even worse result than the one that can be achieved by choosing 1). But we instead think that this option is worth further investigation, since developing an anti-realist noetic account of scientific progress, which relies on a welldefended anti-realist view of understanding, as e.g. the one recently provided by de Regt and Gijsbers (2017), ${ }^{29}$ may be the best way to emphasize the several interesting features that the noetic account displays, and let it spread all its theoretical fertility without having to struggle with the conundrums arising from the adoption of possible-worlds modal realism.

\section{References}

Baumberger, C., Beisbart, C., Brun, G. (2017). What is Understanding? An Overview of Recent Debates in Epistemology and Philosophy of Science. In: Grimm, S., Baumberger, C., Ammon, S. (eds.), Explaining Understanding. New Perspectives from Epistemology and Philosophy of Science. New York: Routledge: 1-34.

Barrett, J.A. (2003). Are Our Best Physical Theories Probably and/or Approximately True? Philosophy of Science, 70: 1206-1218.

Barrett, J.A. (2008). Approximate Truth and Descriptive Nesting. Erkenntnis, 68: 213-224.

Bird, A. (2007). What is Scientific Progress? Noûs, 41: 64-89.

Bueno, O. (2017). The Epistemology of Modality and the Epistemology of Mathematics. In: Fischer, B., Leon, F. (eds.), Modal Epistemology After Rationalism. Cham: Springer: 67-83.

Bueno, O, Shalkowski, S. (2004). Modal Realism and Modal Epistemology: A Huge Gap. In: Weber, E., De Mey, T. (eds.), Modal Epistemology. Brussels: Koninklijke Vlaamse Academie van Belgie: 93-106.

Calude, C.S., Longo, G. (2016). The Deluge of Spurious Correlations in Big Data. Foundations of Science, DOI: 10.1007/s10699-016-9489-4.

Cellucci, C. (2013). Rethinking Logic. Logic in Relation to Mathematics, Evolution, and Method. Dordrecht: Springer.

Cellucci, C. (2017). Rethinking Knowledge. The Heuristic View. Dordrecht: Springer.

Chakravartty, A. (2015). Scientific Realism. In: Zalta, E.N. (ed.), The Stanford Encyclopedia of Philosophy, URL: <http://plato.stanford.edu/archives/fall2015/entries/scientific-realism/>.

Chang, H. (2007). Scientific Progress: Beyond Foundationalism and Coherentism. Royal Institute of Philosophy Supplement, 61: 1-20.

Dellsén, F. (2016a). Scientific Progress: Knowledge versus Understanding. Studies in History and Philosophy of Science, 56: 72-83.

Dellsén, F. (2016b). Understanding without Justification or Belief. Ratio, DOI: $10.1111 /$ rati.12134.

29 There is no space here to adequately argue for this claim, but we think that the noetic account may well be construed in anti-realist terms, and that a promising way for developing this conception is relying on the notion of 'effectiveness' proposed by de Regt and Gijsbers (2017). They propose to replace the usual truth requirement for understanding with an effectiveness condition on understanding, according to which understanding requires representational devices that are scientifically effective, where being 'scientifically effective' means being able to produce useful scientific outcomes such as correct predictions, successful applications and fertile ideas. 
de Regt, H.W. (2009). Understanding and Scientific Explanation. In: de Regt, H.W., Leonelli, S., Eigner, K. (eds.), Scientific Understanding. Philosophical Perspectives. Pittsburgh: Pittsburgh University Press: 21-42.

de Regt, H.W. (2015). Scientific Understanding: Truth or Dare? Synthese, 192, 12: 3781-3797.

de Regt, H.W., Leonelli, S., Eigner, K. (2009). Focusing on Scientific Understanding. In: de Regt, H.W., Leonelli, S., Eigner, K. (eds.), Scientific Understanding. Philosophical Perspectives. Pittsburgh: Pittsburgh University Press: 1-17.

de Regt, H.W., Gijsbers, V. (2017). How False Theories Can Yield Genuine Understanding. In: Grimm, S.R., Baumberger, C., Ammon, S. (eds.), Explaining Understanding. New Perspectives from Epistemology and Philosophy of Science. New York: Routledge: 50-75.

Einstein, A. (1956). On the Movement of Small Particles Suspended in a Stationary Liquid Demanded by the Molecular Kinetic-Theory of Heat. In: Idem, Investigations on the Theory of the Brownian Movement. New York: Dover: 1-18. Original Edition: Idem, (1905). Über die von der molekularkinetischen Theorie der Wärme geforderte Bewegung von in ruhenden Flüssigkeiten suspendierten Teilchen. Annalen der Physik, 322: 549-560.

Elgin, C. (2007). Understanding and the Facts. Philosophical Studies, 132: 33-42.

Elgin, C. (2009). Is Understanding Factive? In: Haddock, A., Millar, A., Pritchard, D. (eds.), Epistemic Value. New York: Oxford University Press: 322-330.

Grimm, S.R. (2006). Is Understanding a Species of Knowledge? The British Journal for the Philosophy of Science, 57: 515-535.

Ippoliti, E. (2014). Generation of Hypotheses by Ampliation of Data. In: Magnani, L. (ed.), Model-Based Reasoning in Science and Technology. Theoretical and Cognitive Issues. Berlin: Springer: 247-262.

Ippoliti, E., Cellucci, C. (2016). Logica. Milano: Egea.

Khalifa, K. (2011). Understanding, Knowledge, and Scientific Antirealism. Grazer Philosophische Studien, 83: 93-112.

Kuhn, T.S. (1970). The Structure of Scientific Revolutions (2 ${ }^{\text {nd }}$ ed.). Chicago: University of Chicago Press.

Kvanvig, J.L. (2003). The Value of Knowledge and the Pursuit of Understanding. New York: Cambridge University Press.

Kvanvig, J.L. (2009). Responses to Critics. In: Haddock, A., Millar, A., Pritchard, D. (eds.), Epistemic Value. New York: Oxford University Press: 339-353.

Laudan, L. (1977). Progress and its Problems. London: Routledge.

Laudan, L. (1981). A Confutation of Convergent Realism. Philosophy of Science, 48, 19-48.

Lewis, D. (1986). On the Plurality of Worlds. Oxford: Blackwell.

Macías, A., Camacho, A. (2008). On the Incompatibility between Quantum Theory and General Relativity. Physics Letters B, 663: 99-102.

Malament, D. (1986a). Gravity and Spatial Geometry. In: Barcan Marcus, R., Dorn, G.J.W., Weingartner, P. (eds.), Logic, Methodology and Philosophy of Science VII. Amsterdam: Elsevier: 405-411.

Malament, D. (1986b). Newtonian Gravity, Limits, and the Geometry of Space. In: Colodny, R. (ed.), From Quarks to Quasars: Philosophical Problems of Modern Physics. Pittsburgh: University of Pittsburgh Press: 181-201.

Mizrahi, M. (2012). Idealizations and Scientific Understanding. Philosophical Studies,160, $237-$ 252.

Morganti, M. (2016). Naturalism and Realism in the Philosophy Science. In: Clark, K.J. (ed.), The Blackwell Companion to Naturalism. Oxford: Blackwell: 75-90.

Niiniluoto, I. (2015a). Optimistic Realism about Scientific Progress. Synthese, DOI: 10.1007/s11229-015-0974-z.

Niiniluoto, I. (2015b). Scientific Progress. In: Zalta, E.N. (ed.), The Stanford Encyclopedia of Philosophy, URL: <http://plato.stanford.edu/archives/sum2015/entries/scientific-progress/>.

Papineau, D., (2016). Naturalism. In: Zalta, E.N. (ed.), The Stanford Encyclopedia of Philosophy, URL: <https://plato.stanford.edu/archives/win2016/entries/naturalism/>.

Perrin, J. (1908). Agitation moléculaire et mouvement brownien. Comptes Rendus de l'Académie des Sciences, 146, 967-970.

Popper, K.R. (1963). Conjectures and Refutations. London: Routledge and Kegan Paul. 
Pritchard, D., Turri, J. (2014). The Value of Knowledge. In: Zalta, E.N. (ed.), The Stanford Encyclopedia of Philosophy, URL: <https://plato.stanford.edu/archives/spr2014/entries/knowledge-value/>.

Rancourt, B.T. (2015). Better Understanding Through Falsehood. Pacific Philosophical Quarterly, DOI: $10.1111 /$ papq. 12134.

Rice, C.C. (2016). Factive Scientific Understanding without Accurate Representation. Biology and Philosophy, 31: 81-102.

Rowbottom, D.P. (2010). What Scientific Progress Is Not: Against Bird's Epistemic View. International Studies in the Philosophy of Science, 24: 241-255.

Saatsi, J. (2016). What is Theoretical Progress of Science? Synthese, DOI: 10.1007/s11229-0161118-9.

Sklar, L. (1981). Do Unborn Hypotheses Have Rights? Pacific Philosophical Quarterly, 62: 1729.

Stanford, K. (2003). Pyrrhic Victories for Scientific Realism. Journal of Philosophy: 100, 553572.

Stanford, P.K. (2006). Exceeding Our Grasp. Oxford: Oxford University Press.

Vaidya, A. (2016). The Epistemology of Modality. In: Zalta, E.N. (ed.), The Stanford Encyclopedia of Philosophy, URL: <https://plato.stanford.edu/archives/win2016/entries/modality-epistemology/>.

Williamson, T. (2000). Knowledge and Its Limits. Oxford: Oxford University Press.

Wray, K.B. (2013). Success and Truth in the Realism/Anti-Realism Debate. Synthese, 190: 17191729.

Zagzebski, L. (2001). Recovering Understanding. In: Steup, M. (ed.), Knowledge, Truth, and Duty. New York: Oxford University Press: 235-251. 